\title{
Cleaning graphene using atomic force microscope
}

\author{
Niclas Lindvall, ${ }^{1, a)}$ Alexey Kalabukhov, ${ }^{1,2}$ and August Yurgens ${ }^{1}$ \\ ${ }^{1}$ Department of Microtechnology and Nanoscience, Chalmers University of Technology, \\ SE-41296 Gothenburg, Sweden \\ ${ }^{2}$ Skobeltsyn Institute of Nuclear Physics, Moscow State University, Moscow, Russian Federation
}

(Received 11 January 2012; accepted 18 February 2012; published online 21 March 2012)

\begin{abstract}
We mechanically clean graphene devices using an atomic force microscope (AFM). By scanning an AFM tip in contact mode in a broom-like way over the sample, resist residues are pushed away from the desired area. We obtain atomically flat graphene with a root mean square (rms) roughness as low as $0.12 \mathrm{~nm}$ after this procedure. The cleaning also results in a shift of the charge-neutrality point toward zero gate voltage, as well as an increase in charge carrier mobility. (C) 2012 American Institute of Physics. [http://dx.doi.org/10.1063/1.3695451]
\end{abstract}

\section{INTRODUCTION}

Graphene is a one atom thick hexagonal lattice of $\mathrm{sp}^{2}$-hybridized carbon atoms, which has attracted much attention recently due to its unique properties. ${ }^{1-3}$ By applying an electric field, the charge carriers in graphene can be tuned from holes to electrons, both having zero rest mass and high mobility. The combination of graphene electronic- and mechanical properties is interesting for both fundamental studies and electronic applications. ${ }^{4-9}$ Pristine graphene is charge neutral and exhibits excellent electronic properties. However, charged impurities, surface contaminants, and structural deformation contribute to local doping. This leads to an inhomogeneous charge density, the so called electron-hole puddles ${ }^{10-14}$ and a shift in the charge neutrality point, $V_{\mathrm{D}}$ (Dirac voltage). Impurities from microfabrication and sample handling are inherent in any graphene processing acting as external scattering centers and affecting the devices properties. ${ }^{15-21}$

Several techniques exist for cleaning graphene. Standard cleaning using solvents cannot remove all these residues. ${ }^{15,19-21}$ Most commonly, graphene is cleaned by high temperature annealing in inert, typically $\mathrm{Ar} / \mathrm{H}_{2}$, atmosphere. ${ }^{15,17,19,22,23}$ While this technique is able to remove most of resist residues through desorption, the coupling between the substrate and graphene may increase, leading to mechanical deformation of the graphene. $^{21}$ Also, suspended graphene becomes rippled upon temperature cycling of only $100-200 \mathrm{~K}^{24}$ Both these effects can cause degradation in device performance. Additionally, many substrates cannot sustain high temperature without oxygen atmosphere, which is incompatible with graphene, e.g., ferroelectric barium strontium titanium oxide (BSTO) thin films on strontium titanium oxide (STO) substrates. Charged contaminants adhere very strongly to these substrates due to the polarized surface. This renders graphene on BSTO heavily contaminated and difficult to clean. Another common method is annealing by Joule heating. ${ }^{16,25}$ This technically simple technique can be done in situ in a cryostat. However, graphene is also in this case heated locally to high temperature leading to rippling or even breakage if too much current is applied. Just recently, mechanical cleaning of graphene was suggested. ${ }^{26-28}$

\footnotetext{
a) Author to whom correspondence should be addressed. Electronic mail: niclas.lindvall@chalmers.se.
}

In this work, we use the mechanical cleaning method to obtain clean, atomically smooth graphene. We scan the tip of an atomic force microscope (AFM) in direct contact with graphene, removing contaminants in broom-like movements. Resist residues are efficiently brushed away, piling up outside the graphene flake. We show that this procedure produces atomically smooth graphene with improved electronic properties.

\section{EXPERIMENT}

We fabricate graphene using mechanical exfoliation on two different kinds of substrates ${ }^{2}$; silicon with $290 \mathrm{~nm}$ thermally grown silicon dioxide and $\mathrm{Nb}$-doped STO substrates with $50 \mathrm{~nm}$ BSTO thin films grown by pulsed laser deposition (PLD). On $\mathrm{SiO}_{2}$, mono- and bilayer graphene are localized using optical microscopes and identified by its optical contrast. ${ }^{29}$ Control samples measured at low temperature and high magnetic fields confirm the number of layers through the quantum Hall effect. ${ }^{4}$ On BSTO, the contrast is significantly smaller and the number of layers is estimated from AFM measurements. Samples are patterned using electron beam lithography (EBL) and subsequent oxygen plasma etching. A second EBL step, followed by evaporation of typically $3 \mathrm{~nm}$ Ti and $60 \mathrm{~nm}$ Au and lift-off, defines electrodes. An overview of studied samples is shown in Table I.

We use AFM in tapping mode to observe the devices both before and after cleaning. In this mode, the AFM does not influence the graphene. Cleaning is done in contact mode using several different AFM probes and different forces. Electrical measurements are performed at room temperature before and after the cleaning. A voltage is applied to the conducting Si substrate acting as a back gate.

\section{RESULTS}

Typical height and phase (inset) images of graphene devices after fabrication are shown in Fig. 1(a). The height rms roughness, $R_{\mathrm{RMS}}$, is $0.77 \mathrm{~nm}$ and $0.47 \mathrm{~nm}$ for a $0.5 \times 0.5 \mu \mathrm{m}^{2}$ area of graphene and bare $\mathrm{SiO}_{2}$ substrate, respectively. We then set the AFM to contact mode, pushing the TiN-coated Si tip in touch with the sample. While the tip is scanned back and forth over the sample, contaminants are mechanically 
TABLE I. Table of samples going through typical fabrication and cleaning procedures. $R_{\mathrm{RMS}}$ is calculated over a $0.5 \times 0.5 \mu \mathrm{m}^{2}$ area.

\begin{tabular}{|c|c|c|c|c|c|c|}
\hline $\begin{array}{l}\text { Sample } \\
\text { name }\end{array}$ & Substrate & $\begin{array}{l}\text { Cleaning } \\
\text { method }\end{array}$ & $\begin{array}{l}\text { Graphene } R_{\mathrm{RMS}} \\
\text { before } \rightarrow \text { after cleaning }(\mathrm{nm})\end{array}$ & $\begin{array}{l}\mathrm{V}_{\mathrm{D}} \text { before } \rightarrow \text { after } \\
\text { cleaning }(\mathrm{V})\end{array}$ & $\begin{array}{l}\text { Mobility before } \rightarrow \text { after } \\
\text { cleaning }\left(\mathrm{cm}^{2} / \mathrm{Vs}\right)\end{array}$ & $\begin{array}{l}\text { Step height } \\
(\mathrm{nm})\end{array}$ \\
\hline S1 & Wet $\mathrm{SiO}_{2} / \mathrm{Si}$ & Hard & $0.50 \rightarrow 0.25$ & - & - & $\sim 1$ \\
\hline S2 & Wet $\mathrm{SiO}_{2} / \mathrm{Si}$ & Hard & $0.77 \rightarrow 0.28$ & - & - & $0.6-0.7$ \\
\hline S3 & Wet $\mathrm{SiO}_{2} / \mathrm{Si}$ & Soft & $0.30 \rightarrow 0.12$ & $+12 \rightarrow-3$ & $4300 \rightarrow 7700$ & $0.5-0.6$ \\
\hline S4 & Wet $\mathrm{SiO}_{2} / \mathrm{Si}$ & Soft & $0.45 \rightarrow 0.13$ & $+24 \rightarrow+2$ & $1200 \rightarrow 1800$ & 0.6 \\
\hline S5 & Dry $\mathrm{SiO}_{2} / \mathrm{Si}$ & Hard & $0.65 \rightarrow 0.21$ & $+20 \rightarrow-10$ & $1400 \rightarrow 1800$ & 0.6 \\
\hline S6 & Dry $\mathrm{SiO}_{2} / \mathrm{Si}$ & Hard & $0.30 \rightarrow 0.21$ & $+16 \rightarrow-1$ & $4200 \rightarrow 5000$ & 0.7 \\
\hline S7 & BSTO / STO & Hard & $1.82 \rightarrow 0.17$ & - & - & 0.5 \\
\hline S8 & BSTO / STO & Hard & $1.60 \rightarrow 0.17$ & - & - & 0.5 \\
\hline
\end{tabular}
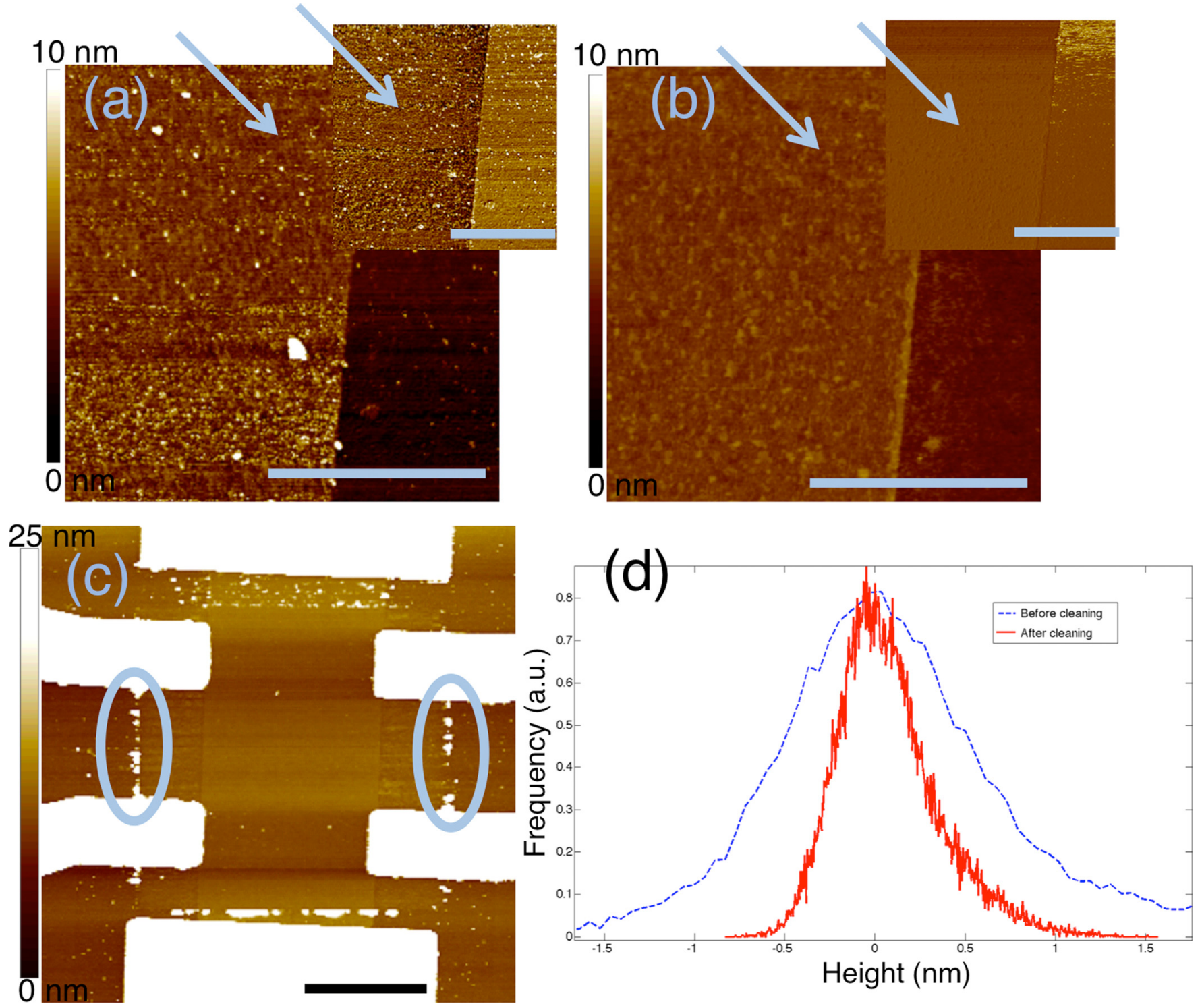

FIG. 1. (Color online) (a) Tapping mode AFM height measurement before cleaning. The inset shows the corresponding phase image. The surface is heavily contaminated after fabrication as seen in both height and phase images. The height rms roughness, $R_{\mathrm{RMS}}$, is $0.77 \mathrm{~nm}$ and $0.47 \mathrm{~nm}$ for a $0.5 \times 0.5 \mu \mathrm{m}^{2}$ area of graphene and bare $\mathrm{SiO}_{2}$ substrate, respectively. (b) Tapping mode measurement of the same area as in (a) after contact mode cleaning in AFM. $R_{\mathrm{RMS}}$ is now $0.28 \mathrm{~nm}$ and $0.29 \mathrm{~nm}$ for graphene and bare $\mathrm{SiO}_{2}$ substrate, respectively. The arrows in (a) and (b) are pointing at the graphene. The scale-bars in (a) and (b) are all $1 \mu \mathrm{m}$. (c) Large-area tapping mode AFM height measurement of a graphene Hall-bar where the central part has been cleaned. The two ellipses point out beads with removed contaminants. The scale-bar is $2 \mu \mathrm{m}$ and the Z-scale is $25 \mathrm{~nm}$. (d) Histogram of surface roughness from before (blue, dashed line) and after (red, solid line) cleaning, respectively. 
pushed to the sides in a broom-like way. Typically three to five such scans are performed. The graphene is generally clean after only two scans and only minor improvement is seen after subsequent cleaning. In Fig. 1(b) the same area as in Fig. 1(a) is shown after four scans in contact mode. $R_{\mathrm{RMS}}$ is now reduced to $0.28 \mathrm{~nm}$ and $0.29 \mathrm{~nm}$ for graphene and bare $\mathrm{SiO}_{2}$ substrate, respectively.

An overview AFM image is shown in Fig. 1(c). The central area of the device is cleaned in contact mode. Around it, there are beads with pushed-away contaminants, as indicated by the two ellipses. The surface is significantly rougher outside the cleaned area. A histogram of the surface roughness before and after cleaning is shown in Fig. 1(d). The distribution becomes much more narrower after the cleaning.

Devices are fabricated on $\mathrm{SiO}_{2}$ grown thermally using both wet- and dry oxidation. We measure $R_{\mathrm{RMS}}$ for both the bare substrate and areas covered with graphene. While $R_{\mathrm{RMS}}$ for pristine "dry" oxide is slightly less than that of "wet", both show a wide range of $R_{\mathrm{RMS}} \sim 0.35-1.40 \mathrm{~nm}$ after fabrication. Corresponding values for graphene after fabrication are $R_{\mathrm{RMS}}$

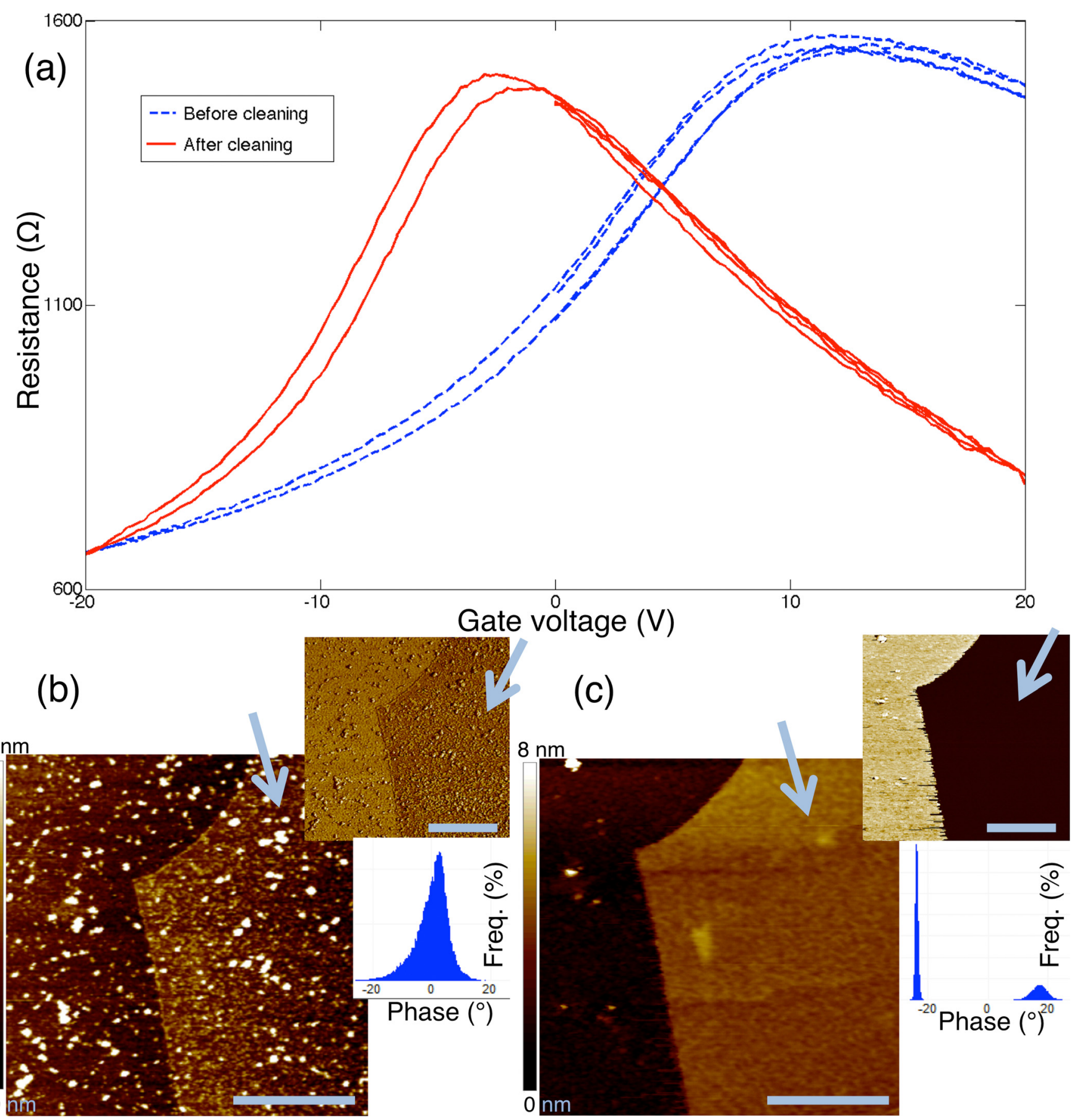

FIG. 2. (Color online) (a) Resistance as a function of gate before (red, solid line) and after (blue, dashed line). The charge neutrality point moves toward zero after cleaning and the estimated mobility increases from $\sim 4300 \mathrm{~cm}^{2} / \mathrm{Vs}$ to $\sim 7700 \mathrm{~cm}^{2} / \mathrm{Vs}$. The Z-scale is $8 \mathrm{~nm}$. (b) and (c) AFM height images before and after cleaning of graphene on BSTO, respectively. The heavy contamination is removed and the atomic steps in the BSTO are clearly seen, including through graphene. Upper insets: AFM phase images. The phase response of both substrate and graphene is almost flat after cleaning, evident from the phase histograms (lower insets). All scale-bars are $500 \mathrm{~nm}$. 
$\sim 0.30-0.65 \mathrm{~nm}$. After cleaning, we obtain $R_{\mathrm{RMS}} \sim 0.25-0.33$ $\mathrm{nm}$ and $R_{\mathrm{RMS}} \sim 0.18-0.23 \mathrm{~nm}$ for wet and dry oxides, respectively. Corresponding values for graphene after cleaning are $R_{\mathrm{RMS}} \sim 0.12-0.25 \mathrm{~nm}$ for both types of oxide. The roughness of graphene depends more on the force applied in the AFM during cleaning than the type of oxide substrate. By using a stiff cantilever (NSG10/TiN, spring constant $C \sim 15 \mathrm{~N} / \mathrm{m}$ ) and a large contact force of $\sim 180 \mathrm{nN}$, the graphene is pushed down toward the substrate leading to similar roughness measurements of both bare $\mathrm{SiO}_{2}$ and graphene $\left(R_{\mathrm{RMS}} \sim 0.2 \mathrm{~nm}\right)$. If a softer cantilever (PPP-CONPt-20, $C \sim 0.17 \mathrm{~N} / \mathrm{m}$ ) and smaller contact force of $\sim 30 \mathrm{nN}$ are used, the graphene shows a significantly lower $R_{\mathrm{RMS}}$ than the bare substrate. Typical values are $R_{\mathrm{RMS}}=0.13 \mathrm{~nm}$ for graphene and $R_{\mathrm{RMS}}=0.33$ nm for bare $\mathrm{SiO}_{2}$, respectively.

The measured step height corresponding to graphene increases after microfabrication to around 1.5-2.0 nm. After cleaning it reduces to $0.6-0.7 \mathrm{~nm}$, typical for clean graphene on $\mathrm{SiO}_{2}$. 30

Several samples are measured electrically before and after cleaning. Resistance is measured as a function of gate voltage $V_{\mathrm{g}}$ applied to the Si substrate. We observe consistent changes in the charge neutrality point, $V_{\mathrm{g}}=V_{\mathrm{D}}$, after cleaning. $V_{\mathrm{D}}$ is zero for ideal un-doped graphene. After fabrication, our devices show positive $V_{\mathrm{D}}$ in the range $12-24 \mathrm{~V}$, indicating $p$-type doping. After cleaning, $V_{\mathrm{D}}$ is found to shift to slightly negative voltages, indicating a weak electron doping probably induced by charges trapped in $\mathrm{SiO}_{2}$. In almost all cases, we also see an increased mobility after cleaning. However, the increase is only significant when using a small contact force while cleaning. In Fig. 2(a), typical resistance measurements with a small contact force are shown. $V_{\mathrm{D}}$ shifts from $+12 \mathrm{~V}$ to $-3 \mathrm{~V}$ while the mobility, estimated from the hole-branch of the curve, increases from $\sim 4300$ $\mathrm{cm}^{2} / \mathrm{Vs}$ to $\sim 7700 \mathrm{~cm}^{2} / \mathrm{Vs}^{31}$

BSTO / STO substrates with graphene devices are heavily contaminated after processing. Due to the polarization of the ferroelectric film, the contaminants are strongly adhered to the surface and are inherently difficult to remove. Height and phase (inset) measurements of graphene on BSTO after microfabrication are shown in Fig. 2(a). Many different solvents together with mild heating and UV-exposure have been tried without success. With mechanical cleaning, it is possible to remove these well-adhered contaminants and recover atomically smooth graphene and bare BSTO substrate. In Figs. 2(b) and 2(c), a graphene sample on BSTO is shown before and after cleaning, respectively. The graphene becomes atomically flat after cleaning, with clearly visible atomic steps in the BSTO. $R_{\mathrm{RMS}}$ reduces from $1.82 \mathrm{~nm}(1.66$ $\mathrm{nm})$ to $0.170 \mathrm{~nm}(0.410 \mathrm{~nm})$ for graphene (BSTO substrate). The phase response changes from a broad distribution to two distinct peaks at roughly $-25^{\circ}$ and $+20^{\circ}$, corresponding to graphene and BSTO, respectively.

\section{CONCLUSIONS}

Mechanical cleaning of graphene using contact mode AFM is an easy way to obtain clean and atomically flat graphene after microfabrication. It improves the charge neutrality of graphene and, using moderate contact force, increases the mobility. This technique appears to be particularly indispensible in the case of graphene devices on ferroelectric BSTO. Despite its low throughput, we believe this strengthens the development of mechanical cleaning methods for graphene.

\section{ACKNOWLEDGMENTS}

Financial support from the Swedish Research Council and the Swedish Foundation for Strategic Research is greatly appreciated. Cleanroom processing was performed using equipment funded by the Knut and Alice Wallenberg Foundation.

${ }^{1}$ K. S. Novoselov, A. K. Geim, S. V. Morozov, D. Jiang, Y. Zhang, S. V. Dubonos, I. V. Grigorieva, and A. A. Firsov, Science 306, 666 (2004).

${ }^{2}$ K. S. Novoselov, D. Jiang, F. Schedin, T. J. Booth, V. V. Khotkevich, S. V. Morozov, and A. K. Geim, Proc. Natl. Acad. Sci. U.S.A. 102, 10451 (2005).

${ }^{3}$ A. K. Geim and K. S. Novoselov, Nature Mater. 6, 183 (2007).

${ }^{4}$ Y. B. Zhang, Y. W. Tan, H. L. Stormer, and P. Kim, Nature 438, 201 (2005).

${ }^{5}$ A. K. Geim, Science 324, 1530 (2009).

${ }^{6}$ S. Bae et al., Nature Nanotech. 5, 574 (2010).

${ }^{7}$ J. Svensson et al., Nano Lett. 11, 3569 (2011).

${ }^{8}$ J. Sun, N. Lindvall, M. T. Cole, K. B. K. Teo, and A. Yurgens, Appl. Phys. Lett. 98, 252107 (2011).

${ }^{9}$ M. Tarasov, N. Lindvall, L. Kuzmin, and A. Yurgens, JETP Lett. 94, 329 (2011).

${ }^{10}$ S. Adam, E. H. Hwang, V. M. Galitski, and S. Das Sarma, Proc. Natl. Acad. Sci. U.S.A. 104, 18392 (2007).

${ }^{11}$ J. Martin, N. Akerman, G. Ulbricht, T. Lohmann, J. H. Smet, K. Von Klitzing, and A. Yacoby, Nature Phys. 4, 144 (2008).

${ }^{12}$ E. A. Kim and A. H. C. Neto, Europhys. Lett. 84, (2008).

${ }^{13}$ J. H. Chen, C. Jang, S. Adam, M. S. Fuhrer, E. D. Williams, and M. Ishigami, Nature Phys. 4, 377 (2008).

${ }^{14}$ Y. B. Zhang, V. W. Brar, C. Girit, A. Zettl, and M. F. Crommie, Nature Phys. 5, 722 (2009).

${ }^{15}$ Y. P. Dan, Y. Lu, N. J. Kybert, Z. T. Luo, and A. T. C. Johnson, Nano Lett. 9, 1472 (2009).

${ }^{16}$ K. I. Bolotin, K. J. Sikes, Z. Jiang, M. Klima, G. Fudenberg, J. Hone, P. Kim, and H. L. Stormer, Solid State Commun. 146, 351 (2008).

${ }^{17}$ X. Du, I. Skachko, A. Barker, and E. Y. Andrei, Nature Nanotech 3, 491 (2008).

${ }^{18}$ F. Chen, J. L. Xia, D. K. Ferry, and N. J. Tao, Nano Lett. 9, 2571 (2009).

${ }^{19}$ M. Ishigami, J. H. Chen, W. G. Cullen, M. S. Fuhrer, and E. D. Williams, Nano Lett. 7, 1643 (2007).

${ }^{20}$ A. Reina, H. Son, L. Jiao, B. Fan, M. S. Dresselhaus, Z. Liu, and J. Kong, J. Phys. Chem. C 112, 17741 (2008).

${ }^{21}$ Z. Cheng, Q. Zhou, C. Wang, Q. Li, C. Wang, and Y. Fang, Nano Lett. 11, 767 (2011).

${ }^{22}$ C. R. Dean et al., Nature Nanotech. 5, 722 (2010).

${ }^{23}$ A. Nourbakhsh et al., J. Phys. Chem. C 114, 6894 (2010).

${ }^{24}$ W. Z. Bao, F. Miao, Z. Chen, H. Zhang, W. Y. Jang, C. Dames, and C. N. Lau, Nature Nanotech. 4, 562 (2009).

${ }^{25}$ J. Moser, A. Barreiro, and A. Bachtold, Appl. Phys. Lett. 91, 163513 (2007).

${ }^{26}$ Z. Liu, P. Boggild, J. R. Yang, Y. Cheng, F. Grey, Y. L. Liu, L. Wang, and Q. S. Zheng, Nanotechnology 22, 265706 (2011).

${ }^{27}$ R. Jalilian, L. A. Jauregui, G. Lopez, J. F. Tian, C. Roecker, M. M. Yazdanpanah, R. W. Cohn, I. Jovanovic, and Y. P. Chen, Nanotechnology 22, 295705 (2011).

${ }^{28}$ A. M. Goossens, V. E. Calado, A. Barreiro, K. Watanabe, T. Taniguchi, and L. M. K. Vandersypen, Appl. Phys. Lett. 100, 073110 (2012).

${ }^{29}$ P. Blake, E. W. Hill, A. H. C. Neto, K. S. Novoselov, D. Jiang, R. Yang, T. J. Booth, and A. K. Geim, Appl. Phys. Lett. 91, 063124 (2007).

${ }^{30}$ F. Giannazzo, S. Sonde, V. Raineri, G. Patane, G. Compagnini, F. Aliotta, R. Ponterio, and E. Rimini, Phys. Status Solidi C 7(3-4 7), 1251 (2010).

${ }^{31}$ S. Kim, J. Nah, I. Jo, D. Shahrjerdi, L. Colombo, Z. Yao, E. Tutuc, and S. K. Banerjee, Appl. Phys. Lett. 94, 062107 (2009). 